\title{
REGIONALIZAÇÃO DO SANEAMENTO NO ESTADO DE ALAGOAS - UM OLHAR PARA A UNIVERSALIZAÇÃO SOB A PERSPECTIVA DOS DIREITOS HUMANOS À ÁGUA E AO SANEAMENTO
}

\section{REGIONALIZATION OF WATER AND SANITATION SERVICES IN THE STATE OF ALAGOAS - A LOOK TO UNIVERSALIZATION FROM THE PERSPECTIVE OF HUMAN RIGHTS TO WATER AND SANITATION}

\begin{abstract}
Cláudia Yukie Nakamura Troni ${ }^{(1)}$
Engenheira Ambiental e MSc. em Engenharia Civil pela Universidade Federal de Viçosa. MSc. em Planejamento e Análise de Políticas Públicas pela Unesp. Diretora técnica e de projetos da VIVIFICAR Gestão em Saneamento e Ambiente. Atua desde 2009 na elaboração de Planos Municipais de Saneamento Básico e de políticas de saneamento. Marcos Helano Fernandes Montenegro ${ }^{(2)}$

Eng. Civil e MSc. em Eng. Urbana e de Construções Civis pela USP. Coordenador Geral do ONDAS e Diretor Nacional da ABES. É Regulador de Serviços Públicos da Adasa DF desde 2009. Atuou na Secretaria Nacional de Saneamento, no IPT, no SEMASA de Santo André. Foi presidente da CAESB - DF, da CEDAE-RJ, da ASSEMAE e da ABES DF.
\end{abstract}

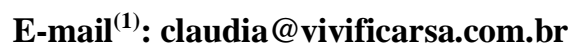

\section{RESUMO}

A Lei $n^{\circ} 14.026 / 2020$ definiu a regionalização do saneamento nos estados como critério para a alocação de recursos públicos federais. O Estado de Alagoas foi o primeiro a leiloar a concessão referente à Região Metropolitana de Maceió (RMM) nos moldes da nova lei o que, de certa maneira, condicionou a regionalização do restante do Estado. A modelagem do estado, ao preservar a RMM, trouxe um desequilíbrio para as Unidades Regionais de Saneamento (URS) dificultando o subsídio cruzado. Ao elaborar o planejamento das URS foram incluídas apenas áreas urbanas e núcleos rurais consolidados de alguns municípios. A universalização, princípio fundamental da LDNSB, ficará ainda mais difícil de ser alcançada, pois grande parte da população sequer foi inserida no planejamento para a concessão, não constará nos contratos e não possuem fontes de recursos definida para provisão dos serviços de abastecimento de água e esgotamento sanitário.

\begin{abstract}
Law no. 14,026/2020 defined the regionalization of water and sanitation services in states as a criterion for the allocation of federal public resources. The State of Alagoas was the first to auction the concession for the Metropolitan Region of Maceió (MRM) along the lines of the new law, which, in a way, conditioned the regionalization of the rest of the State. The modeling of the state, by preserving the MRM, brought an imbalance to the Regional Sanitation Units (RSU) making it difficult to cross-subsidy. When elaborating the planning of the RSU, only urban areas and consolidated rural centers of some municipalities were included. From the point of view of universalization, the fundamental principle of LDNSB, this will become even more difficult to achieve, because a large part of the population has not even been included in the planning for the concession, will not be included in the contracts and do not have sources of resources defined for the provision of water supply and sewage services.
\end{abstract}

Palavras-chave: Regionalização. Saneamento. Alagoas. Direitos Humanos. Privatização.

Key words: Regionalization. Sanitation. Alagoas. Human rights. Privatization.

\section{INTRODUÇÃ̃O}

Diversos processos de regionalização do saneamento foram realizados recentemente no país após a instituição da Lei $n^{\circ}$ 14.026/2020 (BRASIL, 2020) que realizou alterações na Lei $n^{\circ}$ 11.445/2007 - 
Lei de Diretrizes Nacionais de Saneamento Básico (LDNSB) (BRASIL, 2007). Dentre as modificações, foi incluída a obrigação da instituição da regionalização dos serviços públicos de saneamento básico nos estados como critério para a alocação de recursos públicos federais e os financiamentos com recursos da União ou com recursos geridos ou operados por órgãos ou entidades da União (BRASIL, 2020).

Considerando a importância de tais processos, este trabalho objetiva analisar o processo ocorrido no Estado de Alagoas com olhar para a universalização sob a perspectiva dos direitos humanos à água e ao saneamento - cujo reconhecimento se deu pela Assembleia Geral da ONU em julho de 2010 por meio da Resolução 64/292 (UNITED NATIONS, 2010) -, uma vez que o leilão referente à Região Metropolitana de Maceió - capital do Estado - foi o primeiro ocorrido no país nos moldes da nova lei e, de certa maneira, condicionou a regionalização do restante do Estado.

\section{METODOLOGIA}

Para a realização do presente estudo foram pesquisados documentos oficiais disponibilizados nos sites do Governo Federal, do Governo do Estado de Alagoas referentes ao processo de regionalização, bem como análises críticas realizadas sobre o mesmo, em sua maioria, publicados na internet.

Para contextualização e alcance do objetivo proposto, foi realizada uma breve caracterização do Estado de Alagoas e relatado o processo de regionalização, analisando-o e considerando críticas publicadas a respeito do mesmo.

\section{RESULTADOS E DISCUSSÃO}

\subsection{Caracterização do Estado de Alagoas}

O estado de Alagoas localiza-se na Região Nordeste do Brasil. Em 2010, a população no estado era de 3.120.494 habitantes e a estimativa para 2020 era de 3.351 .543 habitantes, sendo o $17^{\circ}$ mais populoso do país (IBGE, 2021). Sua área abrange $27.830,656 \mathrm{~km}^{2}$, com densidade demográfica de $112,33 \mathrm{hab} . / \mathrm{km}^{2}$ (IBGE, 2021). A população rural é estimada em cerca de $26 \%$.

Em termos socioeconômicos, é importante ressaltar que o Índice de Desenvolvimento Humano (IDH) em 2010 era de 0,631, o pior IDH do país (IBGE, 2021). Já o rendimento nominal mensal domiciliar per capita era de $\mathrm{R} \$ 796$ em 2020 , um dos piores do país, estando à frente somente do estado do Maranhão que era de R\$676 no mesmo ano (IBGE, 2021). Dados do Atlas de Desenvolvimento Humano no Brasil referentes ao ano de 2017 (PNUD Brasil, IPEA e FJP, 2020) apontam que 12,39\% da população alagoana era classificada como extremamente pobre, $25,25 \%$ como pobres e $47,51 \%$ como vulneráveis à pobreza.

Em relação ao abastecimento de água em áreas urbanas, Alagoas encontra-se na faixa entre 80 e $90 \%$ de população atendida (SNIS, 2020). Já em relação ao índice de atendimento de população urbana com rede coletora de esgoto, o estado encontra-se na faixa entre $20 \%$ a $40 \%$ (SNIS, 2020).

Em termos comparativos, o percentual de atendimento com rede de abastecimento de água na Região Nordeste era de $73,9 \%$ da população total e de $88,2 \%$ da população urbana. Já o índice de coleta de esgotos era de $82,7 \%$ e 33,7\% do total de esgoto gerado (SNIS, 2020). Conclui-se então que, em relação ao abastecimento de água, Alagoas apresenta percentual de atendimento similar ao de outros estados da Região Nordeste, no entanto, em relação ao esgotamento sanitário encontra-se muito abaixo do índice de atendimento da população urbana para a Região.

\subsection{O processo de regionalização e a concessão da Região Metropolitana de Maceió}


Sob a coordenação do Banco Nacional de Desenvolvimento Econômico e Social - BNDES, foram elaborados estudos de regionalização para o estado de Alagoas, concluídos e apresentados em um evento dessa instituição em 2019, denominado "BNDES com S de Social e Saneamento", com foco à época, na RMM (BNDES, 2019).

Após o advento da Lei $n^{\circ} 14.026 / 2020$, que realizou alterações na Lei no 11.445/2007, o Estado de Alagoas, utilizando a universalização como argumento, elaborou editais para a licitação dos sistemas de abastecimento de água e esgotamento sanitário do estado (ALAGOAS, 2021a) baseando-se nos três blocos regionais sugeridos nos estudos.

Além da Região Metropolitana de Maceió, instituída pela Lei Complementar Estadual no 50/2019, e que, portanto, existia previamente à Lei $\mathrm{n}^{\mathrm{o}}$ 14.026/2020, foram instituídas duas unidades regionais de saneamento básico, através da Lei Ordinária n 8358 de 03 de dezembro de 2020. Desse modo, a adesão à gestão regionalizada era facultativa, devendo ocorrer até os prazos definidos na Lei $\mathrm{n}^{\circ}$ 14.026/2020, de 180 dias após a instituição da unidade regional, que findou em 23 de junho de 2021 (ALAGOAS, 2021b). Importante mencionar que cerca de $90 \%$ dos municípios já haviam aderido à Unidade Regional Agreste Sertão até o final do primeiro prazo estabelecido pelo Estado (ALAGOAS, 2021b), e apenas o município de São Miguel dos Campos havia formalizado a sua não adesão ao convênio. Já para a Unidade Regional Litoral e Zona da Mata, até seis dias antes de findar o prazo para adesão, apenas 15 municípios de um total de 40 havia aderido ao convênio (ALAGOAS, 2021c).

Dentre outras definições, a Lei $n^{\circ}$ 8358/2020 estabeleceu que a representatividade e peso no Órgão Colegiado, parte da estrutura de governança das Unidades Regionais seriam "definidos em Decreto Estadual, de modo objetivo e com base no critério populacional, assegurado ao Estado até $50 \%$ (cinquenta por cento) dos votos" (ALAGOAS, 2020). Tal normativa torna evidente a concentração de poder no Estado em detrimento dos municípios.

Em 07 de maio de 2021 foi publicado então o Decreto $N^{\circ} 74.261$ que dispõe sobre a regulamentação da estrutura de governança das Unidades Regionais de Saneamento Básico da Zona da Mata Litoral Norte e do Agreste Sertão, e dá outras providências.

Importante destacar que o referido decreto estabeleceu para cada Unidade Regional de Saneamento Básico a seguinte composição do Conselho de Desenvolvimento:

I - Representante do Poder Executivo Estadual, cujo voto terá peso 50 (cinquenta);

II - Prefeitos dos Municípios titulares do serviço de saneamento básico que aderiram à Unidade Regional de Saneamento Básico respectiva, cujos votos terão peso conjunto de 40 (quarenta); e

III - (3) três representantes da sociedade civil, todos com direito a voto, que terão peso conjunto de 10 (dez) (ALAGOAS, DECRETO No 74.261/2021d, Art. $3^{\circ}$ ).

Embora o parágrafo $1^{\circ}$ defina que os pesos de cada município serão atualizados conforme a população de cada um, fato que pode ser positivo se balanceado, sua representatividade ainda permanece menor perante o Estado, que deterá peso de $50 \%$ em detrimento de $40 \%$ dos municípios. Ou seja, mesmo que todos os municípios da Unidade Regional sejam contrários a alguma proposição por parte do Estado, os mesmos ainda estarão submetidos à sua decisão, o que não parece ser nada razoável considerando, principalmente, a titularidade dos municípios em relação aos serviços de saneamento básico.

A composição do conselho é ainda mais arbitrária uma vez que, conforme determina o parágrafo $3^{\circ}$ "serão designados pelo Chefe do Poder Executivo do Estado dentre os integrantes de entidades, organizações ou movimentos sociais e populares, ainda que não institucionalizados, visando a 
alcançar a máxima pluralidade e diversidade dos membros do Conselho de Desenvolvimento". Ou seja, serão definidos em última instância, pelo próprio Governo Estadual, ao qual seria dado ainda mais poder. Ainda, não havendo a exigência de ser institucionalizada, oportuniza-se a indicação de entidades não necessariamente representativas de movimentos sociais, podendo ser criadas de forma oportunista para atender objetivos particulares.

O bloco que abrange a Região Metropolitana de Maceió foi leiloado em setembro de 2020 com outorga de mais de R \$ 2,6 bilhões pela BRK Ambiental Participações S.A, sendo o primeiro leilão ocorrido no país após a aprovação da nova lei sobre saneamento. O critério utilizado para julgamento e escolha da melhor proposta foi o de maior valor de pagamento pela outorga, que é criticado por desviar recursos do saneamento e induzir aumento da tarifa, pois o recurso pago a título de outorga terá que ser recuperado e a única forma de remuneração seria pelas tarifas praticadas.

Aguiar (2020) aponta que o primeiro leilão ocorrido incluía justamente o maior percentual do resultado operacional da Companhia Estadual de Alagoas - CASAL, cerca de 95,3\%, já diminuindo as potencialidades de subsídio cruzado que ocorria no Estado. Os demais "lotes" regionais correspondem a uma população de cerca de $53,8 \%$ e a apenas $4,7 \%$ do resultado operacional da empresa, tornando-se muito menos atrativos aos investimentos privados. Carvalho (2021) também ressalta a decisão de agregar no Bloco A a área de maior densidade populacional do estado e afirma que a divisão adotada para a concessão por blocos não foi a mais eficiente, considerando-se que a finalidade básica de tal modelo é o ganho de escala pela associação de municípios de diferentes portes.

Outra questão importante destacada por Aguiar (2020) é que não há definição quanto à destinação dos recursos, ou seja, não há nenhuma garantia de que os recursos obtidos no leilão serão investidos no saneamento dos municípios ainda não licitados, que continuam sendo operados pela CASAL, e que são os que apresentam maiores déficits de atendimento. Acrescenta-se ainda a ausência de garantia de que os investimentos sejam realizados no saneamento dos próprios municípios integrantes da concessão, nas áreas rurais que não constam na área de concessão da licitação realizada e cuja prestação e operação pode ser mais difícil e custosa.

Importante mencionar que alguns termos relativos à Lei Complementar Estadual no 50/2019 foram judicializados, a exemplo da Ação Direta de Inconstitucionalidade (ADI) impetrada pelo Partido Progressistas (PP) em relação ao artigo 14 (MOTA, 2021) e a ADI ajuizada pelo Partido dos Trabalhadores (PT) que questiona a constitucionalidade da inclusão do serviço público de saneamento básico e abastecimento de água como serviços de interesse comum, e também os critérios de composição da Assembleia Metropolitana, ocasionando concentração de poder nas mãos do Estado de Alagoas em detrimento dos municípios (VITAL, 2021).

\subsection{A concessão das Unidades Regionais de Saneamento (URS)}

Em 14 de julho de 2021, o Governo de Alagoas, por meio da Secretaria de Estado de Infraestrutura, abriu consulta pública sobre dois editais de concorrência internacional visando a concessão da prestação regionalizada dos serviços públicos de fornecimento de água e esgotamento sanitário da unidade regional de saneamento Agreste Sertão - Bloco B de Alagoas e Zona da Mata Litoral Norte - Bloco C de Alagoas. O projeto foi estruturado com apoio do Banco Nacional de Desenvolvimento Econômico e Social (BNDES) e o critério de julgamento também será, conforme o leilão já ocorrido, o de maior valor pago pela outorga.

O Bloco B é constituído por 49 municípios, sendo que, em 46 deles os serviços são atualmente prestados pela CASAL, e em 3 municípios os serviços são prestados por autarquias do tipo Serviços Autônomos de Abastecimento de Água e Esgotamento Sanitário - SAAEs (CONSÓRCIO EY / FELSBERG / MUZZI / EMA, 2021a). 
Dados do SNIS (2019) foram utilizados para calcular o índice de atendimento urbano de 91,97\% e índice de atendimento rural de 32,21\% (CONSÓRCIO EY / FELSBERG / MUZZI / EMA, 2021a).

Dentre os municípios objeto do Bloco B de concessão, em 37 deles a área da concessão abrange apenas a área urbana do município e, em apenas 19 deles, constam áreas distintas do distrito sede. Importante ressaltar que o próprio Plano Regional de Saneamento indica que, neste bloco, 45,89\% da população encontra-se em área rural (CONSÓRCIO EY / FELSBERG / MUZZI / EMA, 2021a). Ou seja, o avanço nas condições de saneamento básico não afetará a grande parte da população do bloco.

Nos municípios pertencentes ao Bloco $\mathrm{C}$ de concessão, a prestação em 20 deles é atualmente realizada pela CASAL e em 20 municípios a prestação ocorre por meio de SAAEs (CONSÓRCIO EY / FELSBERG / MUZZI / EMA, 2021b).

Dados do Plano Regional de Saneamento Básico calculados com base no SNIS (2019) para a região apontam índice de atendimento urbano de $81,32 \%$ e índice de atendimento rural de 27,02\% (CONSÓRCIO EY / FELSBERG / MUZZI / EMA, 2021b).

Dentre os municípios objeto do Bloco C de concessão, em 29 deles a área da concessão abrange apenas a área urbana do município e, em apenas 11 deles, constam áreas distintas do distrito sede. Nesse bloco a população rural abrange cerca de $34,9 \%$ da população (CONSÓRCIO EY / FELSBERG / MUZZI / EMA, 2021b). Assim como no Bloco B, grande parte da população não será beneficiada com os avanços nos serviços de saneamento.

A consulta pública aos documentos do edital foi encerrada em 13 de agosto de 2021 (ALAGOAS, 2021e).

Em matéria publicada em novembro de 2021 no site da CASAL foi divulgado que a Companhia está elaborando o Plano de Saneamento Rural, o qual contemplará todas as áreas rurais do estado, no entanto, não foi publicada a previsão de término do mesmo. Pressupõe-se que a mesma ficará responsável pela prestação dos serviços de abastecimento de água e esgoto nos povoados de Alagoas. Há menção a futuros investimentos no fornecimento de água e coleta de esgotos (não mencionam tratamento dos esgotos), mas não são citadas as fontes dos mesmos. No entanto, sem ter contratos de programa com os municípios onde se situam estes povoados a CASAL não pode atuar.

\section{CONCLUSÕES}

A modelagem do Estado, ao preservar a Região Metropolitana, trouxe um desequilíbrio para as Unidades Regionais de Saneamento, dificultando o subsídio cruzado. Ao elaborar o planejamento das URS foram incluídas apenas áreas urbanas e núcleos rurais consolidados de alguns municípios, ou seja, grande parte da população rural não foi incluída na área de concessão embora seja um percentual elevado em relação ao total, principalmente no Bloco B.

A estrutura de governança estabelecida nas legislações em vigor privilegia o Estado em detrimento dos municípios.

A ausência de definição quanto à destinação dos recursos obtidos no leilão da RMM, principalmente quanto ao investimento no saneamento dos municípios ainda não licitados, que continuam sendo operados pela CASAL, e que são os que apresentam maiores déficits de atendimento pode dificultar ainda mais a universalização no Estado, assim como a ausência de garantia de que os investimentos sejam realizados no saneamento dos próprios municípios integrantes da concessão, nas áreas rurais que não constam na área de concessão da licitação realizada e cuja prestação e operação pode ser mais difícil e custosa.

Do ponto de vista da universalização, princípio fundamental da LDNSB, essa ficará ainda mais difícil de ser alcançada, pois grande parte da população sequer foi inserida no planejamento para a 
concessão, não constará nos contratos e não possuem fontes de recursos definida para provisão os serviços de abastecimento de água e esgotamento sanitário.

Foi publicado pela CASAL que o Plano de Saneamento Rural está em elaboração e contemplará toda zona rural do estado de Alagoas, não sendo citada data prevista de término para sua conclusão. Há menção a investimentos em abastecimento de água e coleta de esgotos, mas não são citadas as fontes e nem o prazo para aplicação dos mesmos. Pela matéria, pressupõe-se que a CASAL é quem ficará responsável pela prestação dos serviços nas áreas rurais do estado. No entanto, não existe equacionamento legal para esta prestação de serviço pela CASAL.

Diante de tais fatos, permanecem as seguintes perguntas: quem garantirá o acesso ao saneamento por populações que não constam nas áreas das concessões? Com quais recursos? Como será atingida a universalização nesses casos? O Estado ao elaborar tal planejamento, sem incluir tais áreas, será responsabilizado se não houver o alcance da universalização? A quem cabe essa responsabilidade uma vez que a autonomia municipal é duramente afetada diante de tais definições impostas pela Lei $\mathrm{n}^{\circ} 14026 / 2020 ?$

\section{REFERÊNCIAS}

AGUIAR, Alex M.S. No leilão do saneamento de Maceió os pobres de Alagoas pagam o pato. Disponível em: < https://economia.uol.com.br/colunas/2020/10/09/no-leilao-do-saneamento-demaceio-os-pobres-de-alagoas-pagam-o-pato.htm>. Acesso em: 29 ago. 2021.

ALAGOAS. Decreto No 74.261, de 07 de maio de 2021. Dispõe sobre a regulamentação da estrutura de governança das unidades regionais de saneamento básico da zona da mata litoral norte e do agreste sertão, e dá outras providências. 2021d.

ALAGOAS. Lei $\mathrm{n}^{\circ}$ 8358, de 3 de dezembro de 2020. Institui as unidades regionais de saneamento básico no estado de Alagoas, e dá outras providências.

ALAGOAS. PROGRAMA DE PARCERIA DE INVESTIMENTOS - PPI. Governo de Alagoas abre Consulta Pública para concessão dos serviços de abastecimento de água e de esgotamento sanitário dos Blocos B e C no Estado - Programa de Parcerias de Investimentos. Disponível em: https://www.ppi.gov.br/cpalagoas. Acesso em: 29 ago. 2021 a.

ALAGOAS. SECRETARIA DE ESTADO DA FAZENDA DE ALAGOAS - SEFAZ. 90\% dos municípios do Agreste e Sertão já aderiram à Unidade Regional de Saneamento dentro do prazo. Disponível em: http://www.sefaz.al.gov.br/noticia/item/2907-90-dos-municipios-do-agreste-esertao-ja-aderiram-a-unidade-regional-de-saneamento-dentro-do-prazo. Acesso em: 29 ago. 2021 b.

ALAGOAS. SECRETARIA DE ESTADO DA FAZENDA DE ALAGOAS - SEFAZ. 15

municípios já aderiram a convênio para universalizar água e esgotamento no Litoral e Zona da Mata de Alagoas. Disponível em: http://www.sefaz.al.gov.br/noticia/item/2903-15-municipios-aderem-aconvenio-para-universalizar-agua-e-esgotamento-no-litoral-e-zona-da-mata-de-alagoas. Acesso em: 31 ago. 2021c.

ALAGOAS. SECRETARIA DE ESTADO DA FAZENDA DE ALAGOAS - SEFAZ. Consulta pública para concessão de saneamento se encerra no dia 13 de agosto. Disponível em:

http://www.sefaz.al.gov.br/noticia/item/2940-consulta-publica-para-concessao-de-saneamento-nosblocos-b-e-c-se-encerra-no-dia-13-de-agosto. Acesso em: 29 ago. 2021e.

BANCO NACIONAL DE DESENVOLVIMENTO ECONÔMICO E SOCIAL - BNDES. BNDES com "S" de Social e de Saneamento: Projetos em curso no BNDES. Disponível em: < https://www.bndes.gov.br/wps/wcm/connect/site/f9c48822-4ac5-401d-a745- 
$\underline{S+d e+S o c i a l+e+d e+S a n e a m e n t o . p d f ? M O D=A J P E R E S \& C V I D=m X r Y N S b}>$. Acesso em: 29 out. 2021.

BRASIL. Lei $\mathrm{n}^{\circ} 11.445$, de 05 de janeiro de 2007. Estabelece as diretrizes nacionais para o saneamento básico; cria o Comitê Interministerial de Saneamento Básico; altera as Leis nos 6.766, de 19 de dezembro de 1979, 8.666, de 21 de junho de 1993, e 8.987, de 13 de fevereiro de 1995; e revoga a Lei $\mathrm{n}^{\circ}$ 6.528, de 11 de maio de 1978.

BRASIL. Lei ${ }^{\circ}$ 14.026, de 15 de julho de 2020. Atualiza o marco legal do saneamento básico e altera a Lei n ${ }^{\circ}$ 9.984, de 17 de julho de 2000, para atribuir à Agência Nacional de Águas e Saneamento Básico (ANA) competência para editar normas de referência sobre o serviço de saneamento, a Lei ${ }^{\circ} 10.768$, de 19 de novembro de 2003, para alterar o nome e as atribuições do cargo de Especialista em Recursos Hídricos, a Lei n 11.107, de 6 de abril de 2005, para vedar a prestação por contrato de programa dos serviços públicos de que trata o art. 175 da Constituição Federal, a Lei $\mathrm{n}^{\circ} 11.445$, de 5 de janeiro de 2007, para aprimorar as condições estruturais do saneamento básico no País, a Lei ${ }^{\circ} 12.305$, de 2 de agosto de 2010, para tratar dos prazos para a disposição final ambientalmente adequada dos rejeitos, a Lei $\mathrm{n}^{\circ} 13.089$, de 12 de janeiro de 2015 (Estatuto da Metrópole), para estender seu âmbito de aplicação às microrregiões, e a Lei $\mathrm{n}^{\mathrm{o}} 13.529$, de 4 de dezembro de 2017, para autorizar a União a participar de fundo com a finalidade exclusiva de financiar serviços técnicos especializados.

CARVALHO, Alfredo Assis de. Regulação econômica e contratos sob o novo marco legal do saneamento básico: estudo de caso da concessão da Região Metropolitana de Maceió. 2021. Dissertação (Mestrado em Governança e Desenvolvimento) - Escola Nacional de Administração Pública - ENAP, Brasília, 2021.

CONSÓRCIO EY / FELSBERG / MUZZI / EMA, 2021a. Plano Regional de Saneamento Básico: Abastecimento de Água e Esgotamento Sanitário - Unidade Regional de Saneamento - Bloco B.

CONSÓRCIO EY / FELSBERG / MUZZI / EMA, 2021b. Plano Regional de Saneamento Básico: Abastecimento de Água e Esgotamento Sanitário - Unidade Regional de Saneamento - Bloco C.

Instituto Brasileiro de Geografia e Estatística - IBGE. Panorama. Disponível em: < https://cidades.ibge.gov.br/brasil/al/panorama>. Acesso em: 28 ago. 2021.

MOTA, Ricardo. PP entra com ação no STF para impedir Renan Filho de gastar os R $\$ 2$ bi da Casal. TNH1. 2021. Disponível em: https://blog.tnh1.com.br/ricardomota/2021/06/21/pp-entra-com-acaono-stf-para-impedir-renan-filho-de-gastar-os-r-2-bi-da-casal/. Acesso em: 07 set. 2021.

PNUD Brasil, IPEA e FJP, 2020. Atlas do Desenvolvimento Humano no Brasil. 2020.

Sistema Nacional de Informações sobre Saneamento - SNIS. $25^{\circ}$ Diagnóstico dos Serviços de Água e Esgoto - 2019. Brasília, 2020.

UNITED NATIONS. General Assembly. Resolution A/RES/64/292 adopted by the General Assembly on 28 July 2010. Disponível em: https://www.un.org/ga/search/view_doc.asp?symbol=A/RES/64/292. Acesso em: 24 nov. 2021.

VITAL, Danilo. MACEIÓ X ALAGOAS: STF vai analisar alcance de ingerência estadual em região metropolitana. Consultor Jurídico. Disponível em: https://www.conjur.com.br/2021-jul-04/stfanalisar-ingerencia-estadual-regiao-metropolitana. Acesso em: 20 ago. 2021. 